\title{
NEAR-FIELD INTERFERENCE MICROWAVE DIAGNOSTICS OF CULTURAL PLANTS AND WOOD MATERIALS
}

\author{
Viktor Belichenko*, Andrey Zapasnoy, Aleksandr Mironchev \\ National Research Tomsk State University, Radio Physics Department, 634050 Tomsk, Russia
}

\begin{abstract}
A schematic solution of the near-field interference microwave microscopy technology is discussed. This solution is implemented in the form of a maximally simplified microscope structure. Testing was conducted to determine the capabilities of this microscope. It is shown that technology can be used to solve a number of hygroscopy and defectoscopy problems.
\end{abstract}

\section{Introduction}

In modern devices of near-field microwave microscopy one resonant or nonresonant probe (usually as an open end of coaxial lines of different cross sections) is used for probing [1-3]. The independent studies of several scientific groups have shown that to increase the resolution and sensitivity of near-field microwave microscopy, the so-called "wave" spatial scale should be used, along with "quasistatic" spatial scale (see, for example [4]). However, this conclusions concern to the technology of "one-sided" near-field sounding. At the same time, our study [5] of probing by overlapping near (evanescent) fields of several active probes revealed the presence of a new, "interferential" spatial scale.

\section{The Prototype and the Technique of Experimental research}

One of this work purposes is to confirm given conclusion on the numerical simulation basis using CST Microwave Studio software package. The probing system model includes two identical conical coaxial probes which have $360 \mathrm{~mm}$ long and located coaxially. The apertures of these probes are turned by towards each other. The outer diameter of the probe central conductor in the aperture plane is $30 \mathrm{~mm}$ and the inner diameter of the external conductor is $70 \mathrm{~mm}$; the interval between apertures is equal $L=92 \mathrm{~mm}$. Excitation of the probes is produced by means waveguide ports with identical characteristics. But for the signals on the ports phase shift is introduced.

The real and imaginary parts of the Poynting's vector longitudinal component $P$ were calculated in the interval $(0 \leq z \leq L)$ between apertures of probes, along the axis of their symmetry. Radiated frequency was accepted equal $2 \mathrm{GHz}$ (wavelength $-15 \mathrm{~cm}$ ). The re-

\footnotetext{
* Corresponding author: bvp@mail.tsu.ru
} 
sults of calculations processed with use of the mathematical Mathcad package are presented in the Figure 1. They show the nature of change $\operatorname{Re}(P)$ (Figure $1 a$ ) and $\operatorname{Im}(P)$ (Figure $1 b$ ) depending on initial phase shift $(\Delta \varphi)$ of the fields in the probes: $1-\Delta \varphi=0^{\circ}, 2-$ $\Delta \varphi=15^{\circ}, 3-\Delta \varphi=45^{\circ}, 4-\Delta \varphi=90^{\circ}$.

Directly near the probes apertures there are domains with the maximum values $P$ (module $P$ ). It is interesting to note that, for example, at $\Delta \varphi=0^{\circ}$ oscillating character $P$ is accurately demonstrated. Moreover, there are points in the neighborhood of which $P$ has small values. This circumstance directly indicates the presence of an interference energy flux in the overlapping evanescent fields of the probes. With increase $\Delta \varphi$ values of $\operatorname{Re}(P)$ increase too and the displacement of its maximum in space between apertures of probes occur. For $\operatorname{Im}(P)$ great values near apertures of probes are generally characteristic. However change $\Delta \varphi$ leads to very significant change of $\operatorname{Im}(P)$ localization domain structure. In other words, it is observed considerable redistribution of the stored energy localized near apertures of probes. And the most noted circumstances are observed at $\Delta \varphi=90^{\circ}$.

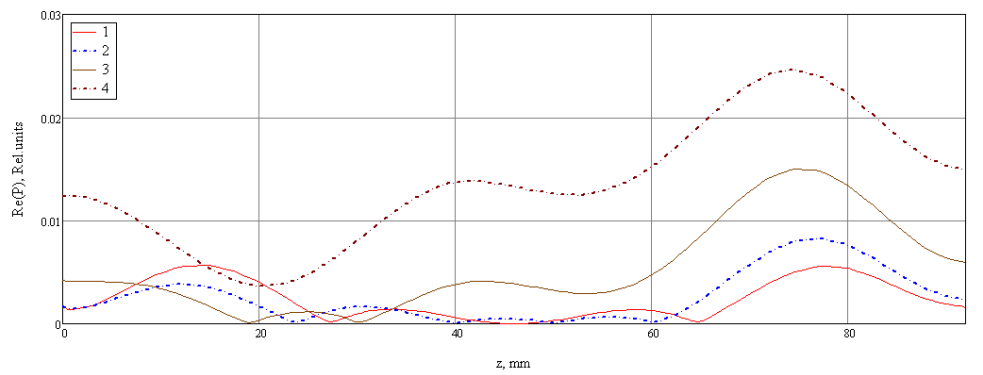

a)

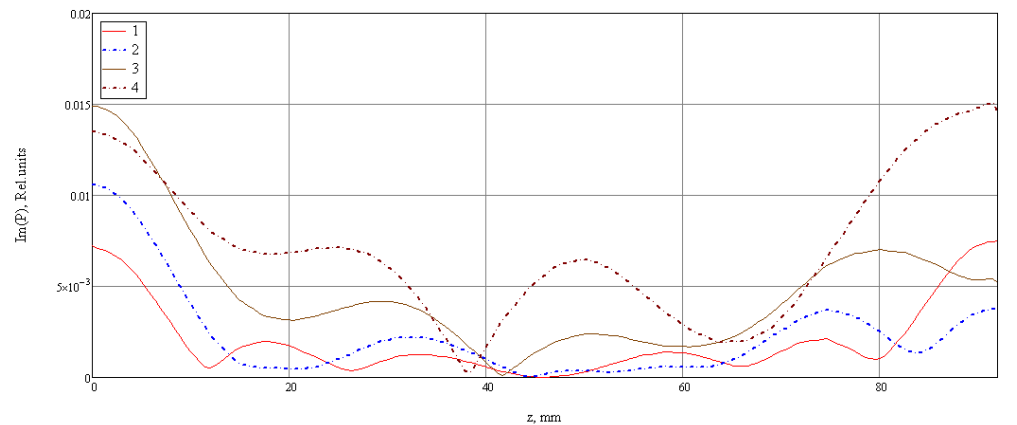

b)

Fig. 1. Distribution of the longitudinal component of complex Poynting's vector in the interval between apertures of two active probes.

It should be emphasized that a change of $\Delta \varphi$ undergoes significant changes and in $\operatorname{Re}(P)$ distribution. Presented here simulation results are quite consistent with the results of [5]. In this paper we prove the existence of additional interference energy flux generated in counter propagating evanescent fields of the two active probes. This energy flux provide a deeper field penetration in the medium under study, as well as improve sensitivity compared to conventional technology "one-sided" sensing. In addition, the detailed theoretical study of the structure of the near-field of a few simple systems radiators has shown that effective control the parameters of said interference energy flows is possible. 
This feasibility has also been confirmed in the present work based on rigorous electrodynamic modeling of the real probing system. It is important to emphasize that the parameters of the interference energy flux formed in the gap between the probe apertures can be controlled; moreover, they can be controlled electrically, namely, by changing the phase difference between the probe fields. This control is realized via "accentuating" one of the interference components of the Pointing vector. This circumstance means that a sufficiently small selected volume of the examined object or medium can be detected via flexible change of the regime of its irradiation by the evanescent fields created by a radiator system (moreover, the motionless system).

\section{Experimental results: analysis and interpretation}

By results of numerical modeling the circuit design of technology of near-field interferential microwave sensing which is most simplified on structure was developed. Based on this circuit design, a working breadboard model of the device was developed and tested experimentally to estimate its resolution and sensitivity.

The breadboard model of the near-field interference microwave microscope contains the following basic elements: a N5230C PNA-L Network Analyzer (Agilent Technologies) with the working frequency band $10 \mathrm{MHz}-40 \mathrm{GHz}$, two coaxial conical horns (probes), power divider, and additional sections of transmission lines (Figure 2). The network analyzer allowed measurements to be performed with high speed and high accuracy, and the correct calibration provided correction for twelve error components.

An operating procedure of the prototype is as follows. A signal generated by the network analyzer splits at the power divider which is working on the frequencies of $10 \mathrm{MHz}-$ $10 \mathrm{GHz}$ with the maximum power of $0.5 \mathrm{~W}$. Then, signals are sent at the inputs of the coaxial conical probes. The probe apertures are faced to each other, the test object is placed in the gap between the apertures and measurements of the reflectivity from the power divider entrance are performed. Creation of required phase shift between the signals arriving on probes is reached due to inclusion in the path of transfer additional sections of coaxial cable, so, that provides the control of interference energy flows which appears in overlapping evanescent fields. The diameter of the probe inner conductor in the aperture plane is 30 $\mathrm{mm}$, the internal diameter of the outer conductor is $70 \mathrm{~mm}$. This provides an opportunity to control the size of overlaping zone of evanescent fields over a wide range using the $10 \mathrm{MHz}-10 \mathrm{GHz}$ microwave radiation, sounding a medium or an object with a counter propagating evanescent fields. The breadboard model of the interference microwave microscope is presented in the Figure 2.
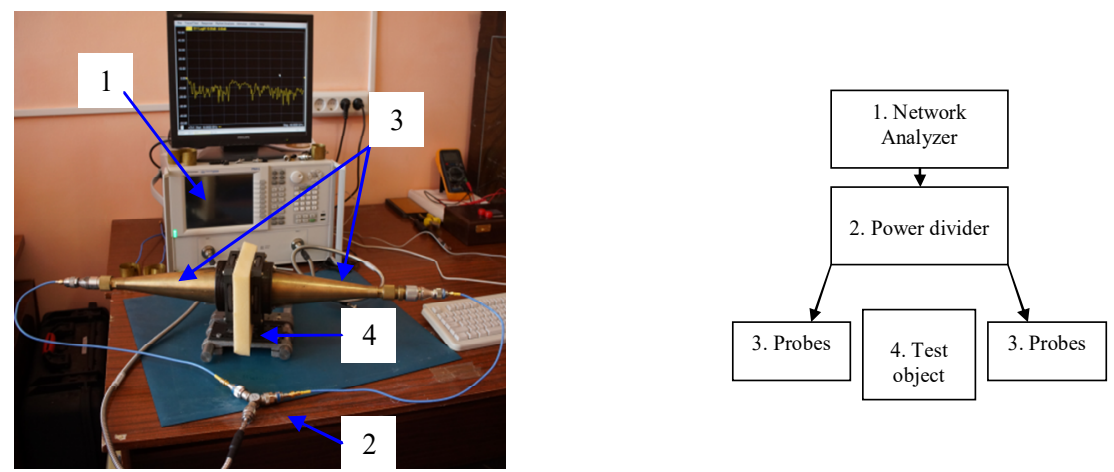

Fig. 2. Breadboard model of the microwave interference microscope. 
As data registered by the network analyzer, the frequency dependence of the complex reflection coefficient (S11) was chosen. The frequency band during measurements was $10 \mathrm{MHz}-10 \mathrm{GHz}$. The step of sampling was $250 \mathrm{kHz}$ since measurements in the chosen frequency band were performed in 38000 points. For convenience of perception during each measurement the registered signals were normalized on the reflectivity for lack of the studied samples. Further we will designate this parameter as the relative reflectivity coefficient (RRC, NS11).

For more detailed analysis of sensitivity of the breadboard model, the series of the experiments concerning researches of quality of seeds of different cultures was conducted. As the studied cultures seeds of soy, flowers of the impatiens and the amaranth were chosen. The purpose of the experiment was to determine the quality of the seeds provided (will they ascend when planting them in the ground or not). Seeds which will sprout have to possess the large supply of water, and, therefore, have big dielectric permittivity and absorb more the radio wave radiation falling on them. For the experiments seeds divided into "fertile" and "infertile" were provided, and the division was carried out by specialists in the botanical garden of the National Research Tomsk State University with the application of a standard methodology.

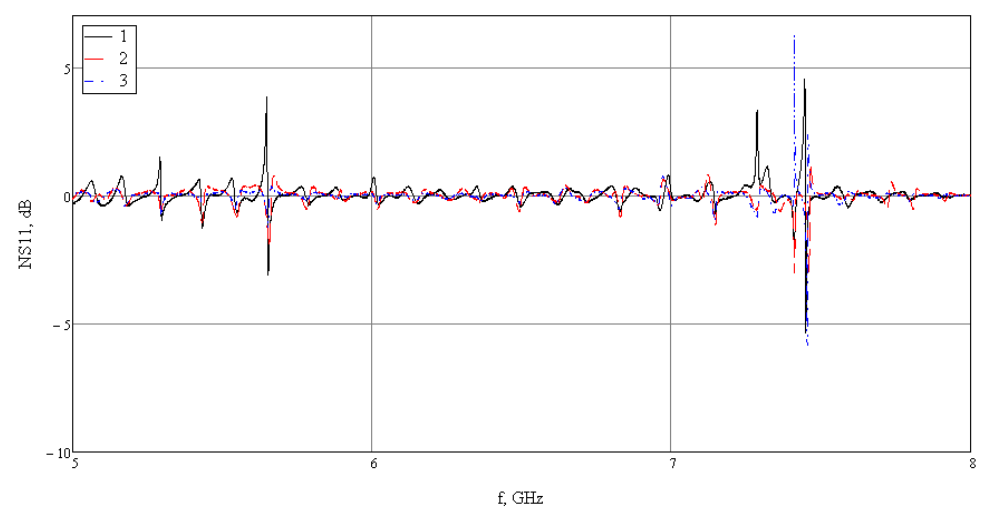

Fig. 3. Frequency dependence of RRC for seeds of three different cultures: 1 - the amaranth, 2 - the impatiens and $3-$ soy.

The experiment was carried out as follows. It was made special the cuvette for seeds which was placed between microscope probes, thickness of the cuvette made $45 \mathrm{~mm}$. After setup and calibration of the breadboard model the measurement data of the empty cuvette were obtained and it date set using for normalization. Then cuvette were filled up in turn by "fertile" and "infertile" seeds, the measurements were normalized and then two signals subtracted in the entire frequency range from $0.5 \mathrm{MHz}$ to $8 \mathrm{GHz}$ (Figure 3) was make. Thus, seeds with maximum RRC were chosen. In Figure 4 is show the frequency band from $2.5 \mathrm{GHz}$ to $3.5 \mathrm{GHz}$, it can be seen that for all three types of seeds, it is possible to allocated seeds with higher dielectric permittivity and, therefore, with a higher likelihood of germination. 


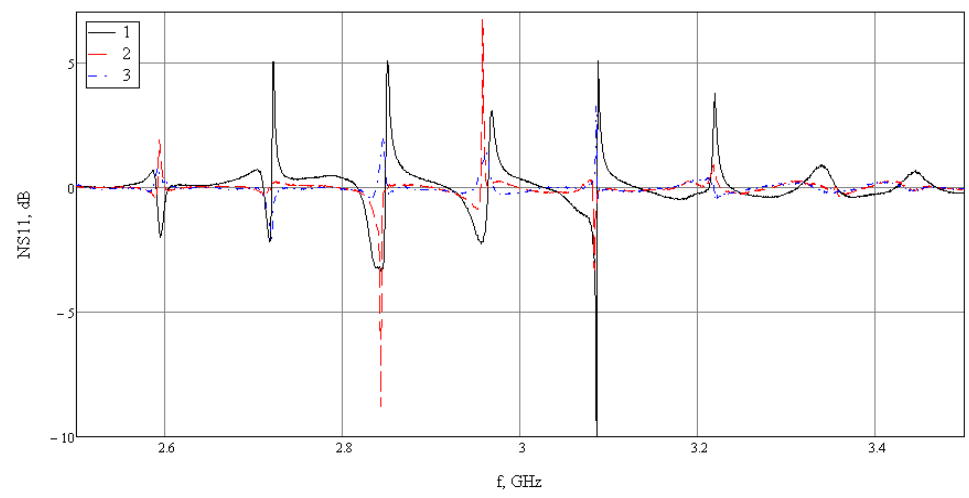

Fig. 4. Frequency dependence of RRC for seeds of three different cultures in the band from $2.5 \mathrm{GHz}$ to 3.5 GHz: 1 - the amaranth, 2 - the impatiens and 3 - soy.

The presented dependences demonstrate that the breadboard model of the microwave interference microscope offered in this work, allows to register even minor changes of moisture content in seeds. As a result of data processing and comparisons of the received results with results these provided specialists of the botanical garden full coincidence is received.

Other series of experiments belonged to clarification of opportunities of the breadboard model at diagnostics of quality of wood materials. The products from the wood having these or those defects including hidden were chosen as objects of researches. As the example in the Figure 5 results of diagnostics of the board of the laminate having defects in the form of through openings with a diameter of 4 and $10 \mathrm{~mm}$ are presented.

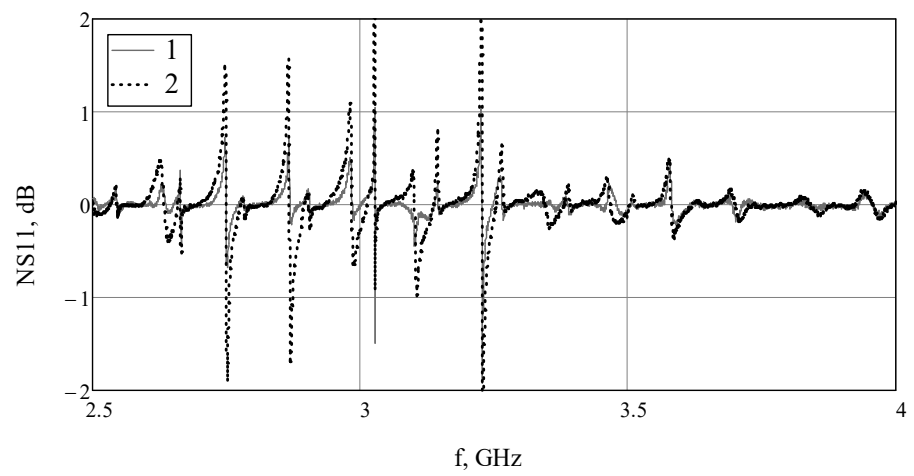

Fig. 5. Frequency dependence of RRC for the laminate $5 \mathrm{~mm}$ thick: 1 - with the opening with a diameter of $4 \mathrm{~mm}, 2$ - with the opening with a diameter of $10 \mathrm{~mm}$.

As we showned, the breadboard model allowed to find similar defects. Also it is showned as with increase in diameter of the opening up to $10 \mathrm{~mm}$ amplitude of the accepted signal also increases. It is essential to emphasize that defect with a diameter of 3-4 mm is fixed even at the frequency $f \approx 2.6 \mathrm{GHz}$ of used the electro-magnetic radiation (at the same time wavelength makes $11.5 \mathrm{~cm}$ ). This demonstrates very essential overcoming the known fundamental limit on permission "in the farfields".

For demonstration differences of traditional technology of sounding from above described we made experiment on diagnostics of defect of the laminate with use evanestsent 
fields only of one probe (Figure 6). The fact that defect existence (even with a diameter of $10 \mathrm{~mm}$ ) is extremely poorly displayed on this schedule attracts attention.

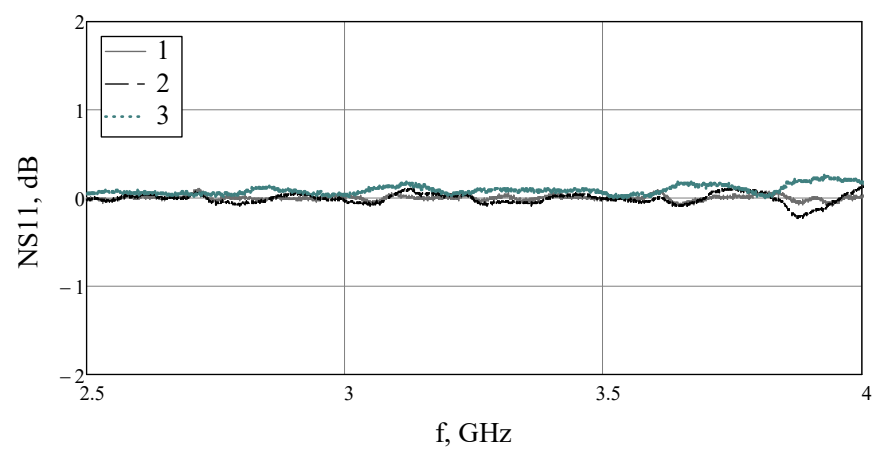

Fig. 6. Frequency dependence of RRC for the laminate $5 \mathrm{~mm}$ thick: 1 - the continuous laminate, 2 - with the opening with a diameter of $3 \mathrm{~mm}, 3$ - with the opening with a diameter of $10 \mathrm{~mm}$.

\section{Conclusion}

The presented results lead to the conclusion that the sensitivity and resolution of described interference microwave microscope are higher in comparison with a microscope constructed according to a conventional scheme. Indeed, our experimental studies, used the most simplified interference microwave microscope model, confirmed this conclusion. In a number of experiments, a microscope with a single probe did not reliably detect the material defect, in contrast to a microscope with two probes. This circumstance is explained by the absence of an additional interference energy flux which generated in the counter propagating evanescent fields of the two probes. This energy flux ensure a deeper penetration of the field into the investigated object, as well as an improvement in sensitivity in comparison with conventional near-field technology. In particular, this effect was manifested in experiments on the hygroscopic properties of seeds of cultural plants, as well as in the detection of defects in wood products.

\section{Acknowledgments}

The research was carried out with the financial support of the Russian Foundation for Basic Research and the Administration of the Tomsk Region within the framework of the scientific project No. 16-42-700942 of the regional competition (r_sibir_a).

\section{References}

[1] S.M. Anlage, V.V. Talanov, A.R. Schwartz, Scanning Probe Microscopy: Electrical and Electromechanical Phenomena at the Nanoscale (Springer-Verlag, New York, 2007)

[2] K.P. Gaikovich, P.K. Gaikovich, Inverse Problems 26, 125013 (2010)

[3] A.N. Reznik, N.V. Yurasova, Technical Physics 51, 86 (2006)

[4] A.N. Reznik, Journal Applied Physics 115, 084501 (2014)

[5] V.P. Belichenko, A.S. Zapasnoy, P.V. Shestakov, Optics and Spectroscopy 118, 633 (2015) 